\title{
ORDER OF THE STANDARD ISOMETRIC MINIMAL IMMERSIONS OF CROSS AS HELICAL GEODESIC IMMERSIONS
}

\author{
By \\ Katsuya MASHIMO
}

\section{Introduction.}

Let $\phi: M \rightarrow \tilde{M}$ be an isometric immersion of a Riemannian manifold $M$ into another Riemannian manifold $\tilde{M}$. Let $\gamma$ be an arbitrary geodesic in $M$ parametrized by arc-length. If the curve $\phi \circ \gamma$ in $\tilde{M}$ is of rank $d$ and has constant curvatures $\kappa_{1}, \cdots, \kappa_{d-1}$ which are independent of the choise of the geodesic $\gamma$, then $\phi: M \rightarrow \tilde{M}$ is called a helical geodesic immersion of order $d$ [7].

The standard isometric minimal immersions of compact rank one symmetric spaces (CROSS) into spheres, which we will define in \$.3, are examples of helical geodesic immersions. In [10] Tsukada calculated the order of the standard isometric minimal immersions of CROSS as helical geodesic immersions except the Cayley projective plane. In this paper we calculate the order of the standard isometric minimal immersions of CROSS in a different manner from the Tsukada's one. Namely we prove the following

THEOREM. The $k$-th standard isometric minimal immersions $\phi_{k}$ of CROSS into spheres are helical geodesic immersions. And the order of the immersions are given as follows;

\begin{tabular}{|c|c|c|}
\hline & $M$ & order of $\phi_{k}$ \\
\hline$S^{n}$ &,$\quad n \geqq 2$ & $k$ \\
\hline$C P^{n}$ &,$\quad n \geqq 2$ & $2 k$ \\
\hline$Q P^{n}$ & , $n \geqq 2$ & $2 k$ \\
\hline Cay $P$ & & $2 k$ \\
\hline
\end{tabular}

From Theorems of the author [5], [6], the order of the standard isometric minimal immersions of CROSS coincide with their degree.

Received December 22, 1982 


\section{Order of helices in a sphere.}

Let $c: I \rightarrow M$ be a curve parametrized by arc-lengrh in a Riemannian manifold $M$. We assume that $\dot{c}(t), \nabla_{t} \dot{c}(t), \cdots, \nabla^{(d-1)}{ }_{t} \dot{c}(t)$ are linearly independent on $I$ and $\dot{c}(t), \nabla_{t} \dot{c}(t), \cdots, \nabla^{(d)}{ }_{t} \dot{c}(t)$ are linearly dependent on $I$. Then we have the usual Frenet-Serret's formula;

$$
\begin{aligned}
& \nabla_{t} \dot{c}(t)=\kappa_{1}(t) Y_{2}(t) \\
& \nabla_{t} Y_{2}(t)=-\kappa_{1}(t) \dot{c}(t)+\kappa_{2}(t) Y_{3}(t), \\
& \nabla_{t} Y_{d-1}(t)=-\kappa_{d-2}(t) Y_{d-2}(t)+\kappa_{d-1}(t) Y_{d}(t), \\
& \nabla_{t} Y_{d}(t)=-\kappa_{d-1}(t) Y_{d-1}(t),
\end{aligned}
$$

where $\kappa_{i}$ are positive functions on $I, Y_{i}$ are orthonormal vector fields along the curve $c$ and $\nabla$ is the Levi-Civita connection of $M$. A curve $c$ is called a helix of order $d$, if $\kappa_{i}$ are constants.

Let $S_{1}^{N}$ be the unit sphere in $\boldsymbol{R}^{N+1}$ centered at the origin. Then $f_{0}(t)=c(t)$ $\cdot c(0), \quad f_{1}(t)=c(t) \cdot \dot{c}(0), \cdots, f_{d}(t)=c(t) \cdot Y_{d}(0)$ satisfies the following differntial equation ([2], p. 177).

$$
\left(\begin{array}{c}
f_{0}^{\prime} \\
f_{1}^{\prime} \\
\cdot \\
\cdot \\
f_{d-1}^{\prime} \\
f_{d}^{\prime}
\end{array}\right)=\left(\begin{array}{cccccc}
0 & -1 & & & & \\
1 & 0 & -\kappa_{1} & & & \\
& \kappa_{1} & 0 & -\kappa_{2} & & \\
& & \cdot & \cdot & \cdot & \\
& & & \cdot & 0 & -\kappa_{d-1} \\
& & & & \kappa_{d-1} & 0
\end{array}\right)\left(\begin{array}{c}
f_{0} \\
f_{1} \\
\cdot \\
\cdot \\
f_{d-1} \\
f_{d}
\end{array}\right)
$$

By a suitable coordinate transformation, a helix in the unit sphere $S_{1}^{N}$ is an orbit of the one parameter subgroup in $S O(N+1)$ generated by the following vector ([2], p. 177).

$$
\left(\begin{array}{cccccccccc}
0 & -1 & & & & & & & \\
1 & 0 & -\kappa_{1} & & & & & & \\
& \kappa_{1} & 0 & -\kappa_{2} & & & & & \\
& & \cdot & \cdot & \cdot & & & & \\
& & & \cdot & 0 & -\kappa_{d-1} & & & \\
& & & \kappa_{d-1} & 0 & 0 & & \\
& & & & 0 & \cdot & \cdot & \\
& & & & & \cdot & 0 & 0 \\
& & & & & & 0 & 0
\end{array}\right)
$$


Then the helix is contained in the totally geodesic submanifold $S_{1}^{d}=\left\{\left(x_{0}, x_{1}, \cdots\right.\right.$, $\left.\left.x_{d}, 0, \cdots, 0\right) \mid \sum_{i=0}^{d}\left(x_{i}\right)^{2}=1\right\}$ and is not contained in any totally geodesic submanifold of $S_{1}^{d}$.

The order of a helix in the unit sphere is characterized by the following

LEMMA 1.1. Let $c: I \rightarrow S_{1}^{N}$ be a helix of order $d$. Then there exists $a$ polynomial $P$ of degree $d+1$ such that

$$
P(d / d t)\left(f_{0}\right)=0, \quad t \in I,
$$

where $f_{0}(t)=c(t) \cdot c(0)$. For any polynomial of which degree is less that $d+1$ (1.3) does not hold.

Proof. Let $P(t)$ be the charasteristic polynomial of (1.2). Then $f_{0}(t)$ satisfies (1.3) ([3], p. 161).

We may assume that $N=d$, i. e., there does not exists any totally geodesic submanifold which contains the helix $c$.

By the differential equation (1.1) we get the following

$$
(d / d t)^{n} f_{0}=a_{n, n} f_{n}+a_{n, n-1} f_{n-1}+\cdots+a_{n, 0} f_{0}, \quad 1 \leqq n \leqq d,
$$

where $a_{n, i}, 0 \leqq i \leqq n$, are constants and $a_{n, n} \neq 0,1 \leqq n \leqq d$. Let $P=\sum_{j=0}^{m} b_{j} x^{m-j}$, $1 \leqq m \leqq d$. Now we assume that (1.3) holds for $P$. Then by (1.3) and (1.4) we get

$$
0=c_{m} f_{m}+c_{m-1} f_{m-1}+\cdots+c_{0} f_{0}
$$

where $c_{m}=a_{m, m}, c_{m-1}, \cdots, c_{0}$ are constants. (1.5) implies that $c(t)$ is contained in the linear subspace which is perpendicular to a non-zero vector $\left(c_{0}, c_{1}, \cdots\right.$, $\left.c_{m}, 0, \cdots, 0\right)$, which is a contradiction. So we get the Lemma. Q.E.D.

REMARK 1.2. (i) The above Lemma is also true, if the parameter $t$ is proportional to the arc-length.

(ii) The polynomial with the smallest degree for which (1.3) holds is unique up to constant factor.

\section{Jaccbi polynomials and their finite cosine expansion.}

Let $p, q$ be real numbers which are greater than -1 . Then for each nonnegative integer $k$ Jacobi polynomial $P_{k}^{(p, q)}(t)$ is defined by

$$
P_{k}^{(p, q)}(t)=\left((p+1)_{k} / k\right)_{2} F_{1}(-k, k+p+q+1 ; p+1 ;(1-t) / 2), \quad t \in \boldsymbol{R},
$$

where ${ }_{2} F_{1}$ is the hypergeometric function of Gauss and $(p+1)_{k}=(p+1) \cdot(p+2) \cdots$ $(p+k)$. The Jacobi polynomials are characterized by the following 
LEMMA 2.1. The Jacobi polynomial $P_{k}^{(p, q)}(t)$ is the unique analytic solution of the following differential equation with $y(1)=1$,

$$
\left(1-t^{2}\right) d^{2} y / d t^{2}-((p+q+2) t+(p-q)) d y / d t+k(p+q+k+1) y=0 \text {. }
$$

When $p=q=n / 2-1\left(n\right.$; positive integer), the Jacobi polynomial $P_{k}^{(p, q)}(\cos \theta)$ has finite cosine expansion.

LEMMA 2.2. ([8], p. 94).

$$
\begin{aligned}
& P_{k}^{(n / 2-1, n / 2-1)}(\cos \theta) \\
&= a_{0} \cos k^{\theta}+a_{1} \cos (k-2) \theta+\cdots+ \\
&\left\{\begin{array}{l}
a_{[k / 2]} \cos \theta, \\
a_{[k / 2]}, \text { if } k \text { is } k \text { is an odd integer },
\end{array}\right.
\end{aligned}
$$

where $a_{j}, 0 \leqq j \leqq[k / 2]$, are positive constants.

We can derive the finite cosine expansion for some Jacobi polynomials by the following

LEMMA 2.3. ([8], p. 250). Let $p, q, r$ be real numbers such that $r \geqq p \geqq 0$, $q>-1$. Then

$$
P_{k}^{(r, q)}(t)=\sum_{j=0}^{k} a_{k, j} P_{j}^{(p, q)}(t)
$$

where $a_{k, j}$ are positive constants.

\section{Proof of the Theorem.}

Let $(G, K)$ be an irreducible symmetric pair of compact type. Let $\mathfrak{g}$ [resp.

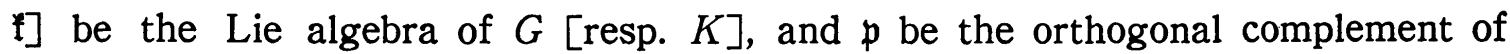
$f$ in $g$ with respect to the Killing form $B$ of $g$. Let $g_{0}$ be the $G$-invariant Riemannian metric on $M=G / K$, which is a $G$-invariant extension of $-\left.B\right|_{p \times p}$. Then $\left(M, g_{0}\right)$ is a Riemannian symmetric space.

Let $V$ be a real irreducible representation of $G$ with a $G$-invariant inner product $\langle$,$\rangle , which contains a K$-fixed unit vector $v_{0}$ (such a representation is called a class one representation of the pair). Then

$$
\phi: M \longrightarrow V ; g K \longrightarrow g \cdot v_{0}
$$

realizes an isometric immersion of $\left(M, c^{2} g_{0}\right)$ for some constant $c$ into the unit sphere in $V$ centered at the origin [11].

Define a linear map $A: V \rightarrow C^{\infty}(M)$ by 


$$
A(v)(g K)=\left\langle v, g \cdot v_{0}\right\rangle, \quad g \in G .
$$

Then $A(v)$ is an eigen-function of the Laplace-Beltrami operator $\Delta$ of $\left(M, g_{0}\right)$ [11]. Actually $A$ is a $G$-homomorphism. Since $\Delta \circ g=g \circ \Delta, \Delta$ is a scalar operator on $A(V)$, i. e., $A(V)$ is contained in some eigin-space of $\Delta$, say the $k$-th eigen-space $V^{k}$. If $M$ is of rank one $A(V)=V^{k}[1]$. So we denote by $\phi_{k}$ the immersion defined by (3.1) when $A(V)=V^{k}$, and call the $k$-th standard isometric minimal immersion of $M$.

Hereafter we assume that $M$ is of rank one. The $v_{0}$ is unique up to sign for each class one representation [1]. It is easily seen that $\phi_{k}$ is a helical geodesic immersion.

Since $v_{0}$ is a $K$-fixed vector $h=A\left(v_{0}\right)$ satisfies

$$
h(k \cdot p)=h(p), \quad k \in K, \quad p \in M .
$$

The function in $V^{k}$ which satisfies (3.2) is called a zonal spherical function of $V^{k}$, which is unique up to sign (since $v_{0}$ is so).

Now we give a explicit expression of $h=A\left(v_{0}\right)$ using Jacobi polynomials.

Assume that a function $h \in C^{\infty}(M)$ satisfies $(3.2)$, then thre exists a function $h_{0}$ defined on $[0, \pi]$, where $\pi$ is the diameter of $\left(M, g_{0}\right)$, such that

$$
h(p)=h_{0}(d(p))
$$

where $d(p)$ is the distance of $p$ from the origin $e K$ on $M$. For such functions there exists a differential operator $\partial(\Delta)$ on $(0, \pi)$ such that

$$
(\Delta h)(p)=\left(\partial(\Delta) h_{0}\right)(d(p)), \quad d(p) \in(0, \pi) .
$$

From $[4$, p. 270$]$ or $[9$, p. 203$], \partial(\Delta)$ is calculated as follows

LEMMA 3.1.

$$
\partial(\Delta)= \begin{cases}-d^{2} / d \theta^{2} & , \text { if } M=S^{1}, \\ -d^{2} / d \theta^{2}-(n-1) \cot \theta d / d \theta & , \text { if } M=S^{n}, n \geqq 2 \\ -d^{2} / d \theta^{2}-((n-1) \cot \theta / 2+\cot \theta) d / d \theta & , \text { if } M=C P^{n}, n \geqq 2, \\ -d^{2} / d \theta^{2}-(2(n-1) \cot \theta / 2+3 \cot \theta) d / d \theta, & \text { if } M=Q P^{n}, n \geqq 2, \\ -d^{2} / d \theta^{2}-(4 \cot \theta / 2+7 \cot \theta) d / d \theta & , \text { if } M=\text { Cay } P^{2} .\end{cases}
$$

After a change of variable $t=\cos \theta, \partial(\Delta)$ is given as follows. 
LEMMA 3.2.

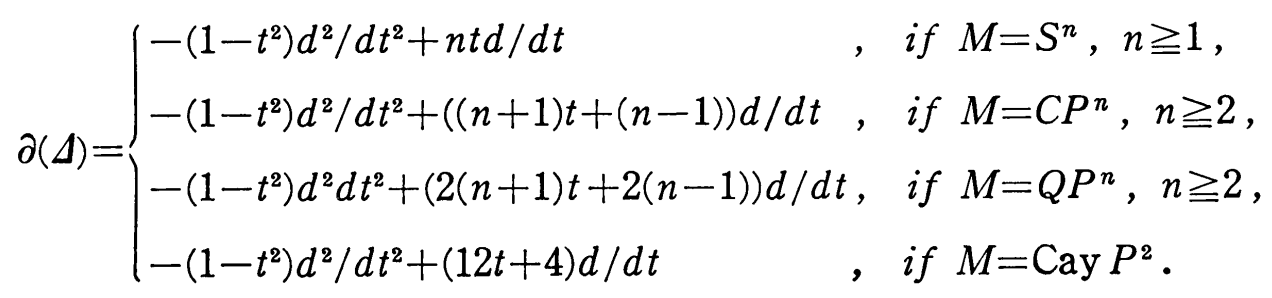

By a formula of Freudenthal [9] we can calculate the eigenvalue of $\Delta$.

LEMMA 3.3. The $k$-th eigen-value of $\Delta$ is

$$
\begin{array}{ll}
k(k+n-1), & \text { if } M=S^{n}, n \geqq 1 \\
k(k+n), & \text { if } M=C P^{n}, n \geqq 2 \\
k(k+2 n+1), & \text { if } M=Q P^{n}, n \geqq 2 \\
k(k+11), & \text { if } M=\text { Cay } P^{2} .
\end{array}
$$

Let $k$ be an eigen-function of $\Delta$ corresponding to the $k$-th eigen-value which satisfies (3.2), Then $h_{0}$ (defined by (3.3)) is an eigen-function of $\Delta$ corresponding to the same eigen-value. So from Lemma 2.1, Lemma 3.2 and Lemma 3.3 we get the following

LEMMA 3.4. Tine zonal spherical function $h$ of $\left(M, g_{0}\right)$ which is contained in $V^{k}$ is $h(p)=h_{0}(d(p))$, where $h_{0}$ is

$$
h_{0}(\theta)= \begin{cases}P_{k}^{(n / 2-1, n / 2-1)}(\cos \theta), & \text { if } M=S^{n}, n \geqq 1, \\ P_{k}^{(n-1,0)}(\cos \theta) & , \text { if } M=C P^{n}, n \geqq 2, \\ P_{k}^{(2 n-1,1)}(\cos \theta) & , \text { if } M=Q P^{n}, n \geqq 2, \\ P_{k}^{\left(7,{ }^{2}\right)}(\cos \theta) & , \text { if } M=\text { Cay } P^{2} .\end{cases}
$$

Let $\gamma_{X}(t)$ be a geodesic in $M$ issuing from the origin $e K$ in the unit direction $X \in T_{e K} M=\mathfrak{p}$. Since $M$ is of rank one (3.2) implies that $A\left(v_{0}\right)\left(\gamma_{X}(t)\right)=\gamma_{X}(t) \cdot \gamma_{X}(0)$ is independent of the choise of $X$. From the uniqueness of the zonal spherical function we get $A\left(v_{0}\right)\left(\gamma_{X}(t)\right)=k h_{0}(t), t \in(0, \pi)$, for some non-zero constant $k$. So we have only to calculate the smallest degree of the polynomial such that (1.3) holds.

CASE 1. $M=S^{n}(n \geqq 2)$. Since $h_{0}$ has finite cosine expansion by Lemma 2.2, $h_{0}$ satisfies (1.3) for 


$$
P(t)=\left(t^{2}+k^{2}\right) \cdot\left(t^{2}+(k-2)^{2}\right) \cdots \cdots\left\{\begin{array}{l}
\left(t^{2}+1\right), \text { if } k \text { is an odd integer, } \\
\left(t^{2}+4\right) \cdot t, \text { if } k \text { is an even integer. }
\end{array}\right.
$$

It is easily verified that (1.3) does not hold for any polynomial of which degree is less that $k+1$. So the Theorem is proved.

CASE 2. $M=C P^{n}(n \geqq 2), M=Q P^{n}(n \geqq 2)$ or $M=$ Cay $P^{2}$. Since $h_{0}$ has finite expansion by Jacobi polynomials from Lemma $2.3, h_{0}$ has finite cosine expansion

$$
h_{0}(\theta)=\sum_{j=0}^{k} a_{j} \cos j \theta,
$$

where $a_{j}$ are positive constants. So $h_{0}$ satisfies (1.3) for

$$
P(t)=\left(\prod_{j=0}^{k}\left(t^{2}+j^{2}\right)\right) \cdot t .
$$

Also in this case it is easily verified that (1.3) does not hold for any polynomial of which degree is less than $2 k+1$. So the Theorem is proved.

\section{References}

[1] Berger, M., Gouduchon, P. et Mazet, E., Le spectre d'une variete Riemannienne, Lecture Notes in Math., 192, Springer Verlag, Berlin-Heidelberg-New York, 1971.

[2] Besse, A., Manifolds all of whose geodesics are closed, Springer Verlag, BerlinHeiderberg-New York, 1978.

[3] Goursat, E., A course in Mathematical Analysis, Vol. 2, Part 2, Differential equations, Dover, New York, 1959.

[4] Harish-Chandra, Spherical functions on semi-simple Lie groups, Amer. J. Math., 80 (1958), 241-310.

[5] Mashimo, K., Degree of the standard isometric minimal immersions of complex projective spaces into spheres, Tsukuba J. Math., 4 (1980), 133-145.

[6] - Degree of the standard isometric minimal immersions of symmetric spaces of rank one into spheres, Tsukuba J. Math., 5 (1981), 291-297.

[7] Sakamoto, K., Helical immersions into a unit sphere, Math. Ann., 261 (1982), 63-80.

[8] Szegö, G., Orthogonal polynomials, Amer. Math. Soc. Colloq. Publication, Vol. 23, Amer. Math. Soc., Providence, RI, 1939.

[9] Takeuchi, M., Modern theory of spherical functions (in Japanese), Iwanami Shoten, Tokyo, 1974.

[10] Tsukada, K., Helical geodesic immersions of compact rank one symmetric spaces, to appear.

[11] Wallach, N., Minimal immersions of symmetric spaces, Symmetric spaces, Marcel Dekker, New York, 1972, 1-40.

Institute of Mathematics

University of Tsukuba

Sakura-mura, Niihari-gun

Ibaraki, 305 Japan 